\title{
Comodulation detection differences in children and adults
}

\author{
Joseph W. Hall III, ${ }^{\text {a) }}$ Emily Buss, and John H. Grose \\ Department of Otolaryngology/Head and Neck Surgery, University of North Carolina School of Medicine, \\ Chapel Hill, North Carolina 27599, USA
}

(Received 1 June 2007; revised 14 November 2007; accepted 8 January 2008)

\begin{abstract}
This study investigated comodulation detection differences (CDD) in children (ages 4.8-10.1 years) and adults. The signal was $30-\mathrm{Hz}$ wide band of noise centered on $2 \mathrm{kHz}$, and the masker consisted of six $30-\mathrm{Hz}$ wide bands of noise spanning center frequencies from 870 to $4160 \mathrm{~Hz}$. The envelopes of the masking bands were always comodulated, and the envelope of the signal was either comodulated or random with respect to the masker. In some conditions, the maskers were gated on prior to the signal in order to minimize effects related to perceptual fusion of the signal and masker. CDD was computed as the difference between signal detection thresholds in conditions where all bands were comodulated and conditions where the envelope of the signal was random with respect to the envelopes of the maskers. Values of CDD were generally small in children compared to adults. In contrast, masking release related to masker/signal onset asynchrony was comparable across age groups. The small CDDs in children are discussed in terms of sensitivity to comodulation as a perceptual fusion cue and informational masking associated with the detection of a signal in a complex background, an effect that is ameliorated by asynchronous onset.
\end{abstract}

(C) 2008 Acoustical Society of America. [DOI: 10.1121/1.2839006]

PACS number(s): 43.66.Dc, 43.66.Ba [RYL]

Pages: 2213-2219

\section{INTRODUCTION}

The detection of a narrow band of noise in the presence of one or more narrow bands of masking noise is often dependent on the relative fluctuation patterns across the noise bands. Threshold differences between conditions where all bands share the same envelope (comodulated) and conditions where envelope differences exist across bands are termed comodulation detection differences (CDD) (Cohen and Schubert, 1987; McFadden, 1987; Wright, 1990). In the present study, we refer to the case where both the masker and signal bands have the same envelope as an A/A condition, and the case where the signal has a fluctuation pattern independent from the maskers as an $\mathrm{A} / \mathrm{B}$ condition. The classical $\mathrm{CDD}$ effect is that the threshold is lower (better) in the A/B condition than in the A/A condition (e.g., Cohen and Schubert, 1987; McFadden, 1987; Wright, 1990). One interpretation of the relatively poor signal detection in the A/A case is based on sound source segregation (Cohen and Schubert, 1987; McFadden, 1987; Wright, 1990). By this account, the common fluctuation pattern across frequency in the A/A condition perceptually fuses the masker and signal, hindering the detection of the signal as a separate auditory object.

Borrill and Moore (2002) and Moore and Borrill (2002) have pointed out that CDD may not necessarily depend upon processes related to sound source segregation, but instead could hinge upon peripheral auditory processes related to masking/suppression. By this account, the coincidence between the energy peaks of the masker and those of the signal make the masking/suppression of the signal particularly efficient. Performing an experiment in which the masker in the

\footnotetext{
a) Author to whom correspondence should be addrdssed. Electronic mail: jwh@med.unc.edu.
}

A/A condition had a leading temporal fringe, Moore and Borrill found that their listeners obtained no improvement in signal threshold with this asynchrony. They pointed out that if poor signal detection in the $\mathrm{A} / \mathrm{A}$ condition were due to perceptual fusion between masker and signal, the powerful sound segregation cue of onset asynchrony should have resulted in a threshold improvement. Their finding of no threshold improvement therefore undermined the perceptual fusion hypothesis and instead provided support for the masking/suppression hypothesis. In a later study by Hall et al. (2006), the results from several listeners were consistent with the findings of Moore and Borrill (2002), with A/A thresholds being similar between conditions where signal and masker were gated synchronously and conditions where the masker had a leading fringe. However, Hall et al. (2006) found that other listeners, who had relatively high thresholds in the synchronous A/A condition, achieved substantially lower thresholds in the asynchronous A/A condition. They speculated that in these listeners, at least part of the CDD effect was not due to peripheral factors but, instead, was related to perceptual fusion. Further conditions tested by Hall et al., where the masker had spectral uncertainty on a trial by trial basis (e.g., Neff and Callaghan, 1987; Neff and Green, 1987; Neff and Callaghan, 1988; Lutfi, 1990; Leek et al., 1991; Kidd et al., 1994, 2002; Richards et al., 2002; Kidd et al., 2003), were also consistent with an interpretation that perceptual fusion factors contributed to at least part of the CDD effect in some listeners. Nevertheless, results of Hall et al. (2006) indicated that the across-frequency difference in fluctuation pattern associated with the A/B conditions was a relatively weak cue for perceptual segregation when contrasted with the cue of onset asynchrony. This is consistent 
with the interpretation of Turgeon et al. (2002) that, compared to asynchrony, comodulation is a relatively weak grouping/segregation cue.

The present study examined CDD for fixed-frequency signal/masker stimuli in children and in adults. There are two related reasons that such a developmental approach might be informative. One is that results should provide further insight into the nature of the CDD phenomenon. If the interpretation of Hall et al. (2006) is correct that at least part of the CDD in some listeners arises from effects related to perceptual fusion but that the associated cues are relatively weak, then it is likely that, compared to adults, CDD in children should be relatively small. This follows because whereas some children appear to enjoy nearly adult-like release from masking for the relatively strong perceptual segregation cue of signal/ masker onset asynchrony (Hall et al., 2005; Leibold and Neff, 2007), there is some evidence that their ability to benefit from less salient cues for masking release can be quite poor in comparison to adults. For example, whereas Hall et al. (2005) found that signal/masker onset asynchrony was associated with substantial release from informational masking in both adults and children, the cue of lateralization via interaural intensity difference resulted in a modest masking release for adults and essentially no masking release for children. Thus, if part of CDD in adult listeners is related to the use of a relatively weak cue for sound source grouping/ segregation, then relatively small CDD might be expected for children. In addition to providing further insight into the nature of CDD, the present approach should improve our understanding of the development of sound source segregation processes. Specifically, the results should allow examination of the idea that the development of sensitivity to perceptual segregation/grouping cues may be prolonged for cues that appear to be relatively weak in adults.

\section{METHODS}

\section{A. Listeners}

There were nine adult listeners ranging in age from 18 to 51 years. There were fifteen children ranging in age from 4.8 to 10.1 years (with a mean of 8.0 years and standard deviation of 2.0 years). Listeners had thresholds of $20 \mathrm{~dB}$ HL (ANSI, 2004) or better between octave frequencies of 0.25 and $8.0 \mathrm{kHz}$.

\section{B. Stimuli}

The signal was a $30-\mathrm{Hz}$ wide band of noise centered on $2 \mathrm{kHz}$. The masker was a set of $30-\mathrm{Hz}$-wide noise bands having center frequencies of $0.870,1.169,1.540,2.570$, 3.280 , and $4.160 \mathrm{kHz}$. These frequencies were the same as those used in our previous CDD experiment on adult listeners (Hall et al., 2006). Thresholds were obtained for both A/A conditions where both the masking and signal bands were comodulated and $\mathrm{A} / \mathrm{B}$ conditions where the envelope of the signal was independent with respect to the envelopes of the comodulated masking bands. Each masking band was presented at a level of $48 \mathrm{~dB}$ SPL. The stimuli were generated in the spectral domain and were converted to the time domain via inverse fast Fourier transform (FFT). The stimuli
TABLE I. Correlations between child age and masked threshold for the A/A condition, A/B condition, and A/A condition with the cue of onset asynchrony. Also shown are correlation between child age and the derived measures of CDD and masking release due to onset asynchrony in the A/A condition. Correlations that were significant $(p<0.05$, two tailed) are noted by an asterisk.

\begin{tabular}{lllllll}
\hline \hline \multicolumn{3}{c}{ Threshold estimates } & & & \multicolumn{2}{l}{ Derived measures } \\
\cline { 6 - 6 } \cline { 5 - 6 } & A/A & A/B & A/A Fringe & & CDD & $\begin{array}{l}\text { Masking release due } \\
\text { to onset asynchrony }\end{array}$ \\
\hline$r$ & -0.51 & $-0.58^{*}$ & $-0.59^{*}$ & & 0.39 & -0.44 \\
$p$ & 0.052 & 0.025 & 0.026 & & 0.150 & 0.120 \\
\hline \hline
\end{tabular}

were played out of one channel of a real time processor (RP2, TDT) at a rate of $24.4 \mathrm{kHz}$, routed to a headphone buffer (HB7, TDT), and presented over the left earphone of a pair of Senheisser headphones (HD 265).

The signal and masking bands were either gated synchronously or were gated such that the masker bands were turned on before the signal band (all bands were gated off together). Gating was accomplished via multiplication with a 50-ms, raised cosine. In the synchronous gating conditions, the signal and masker had a duration of $550 \mathrm{~ms}$, including ramps. In the asynchronous gating conditions, the signal was gated on $200 \mathrm{~ms}$ after the masker, and both signal and masker were fully gated off $550 \mathrm{~ms}$ later. The interstimulus interval was $400 \mathrm{~ms}$ in the synchronous and $200 \mathrm{~ms}$ in the asynchronous condition.

\section{Procedure}

The task used a three-alternative forced-choice procedure with the signal level adjusted in a two-down one-up track estimating the $70.7 \%$ correct point on the psychometric function (Levitt, 1971). The signal level was adjusted in steps of $4 \mathrm{~dB}$ for the first two reversals and then in steps of $2 \mathrm{~dB}$ for the remaining six reversals. Threshold was taken as the average level at the last six reversals. All thresholds were obtained in blocks, by condition, with conditions completed in a random order. Three threshold estimates were obtained in each condition, with a fourth estimate obtained in instances where the first three thresholds varied by more than $3 \mathrm{~dB}$. Listening intervals were marked visually using animation on a video monitor. Over the course of a threshold run, a cartoon picture was revealed, in the form of a jigsaw puzzle, with one piece exposed following each correct response. The cartoon was completely revealed and performed a 2-s animation at the end of the threshold run. All listeners used this method.

\section{RESULTS AND DISCUSSION}

Data of the children were initially analyzed to determine whether the correlations between age and CDD or between age and the masking release due to onset asynchrony in the A/A condition were significant for the group of children tested here. This was done to determine whether the child data should be separated into subgroups by age for comparisons with adult data. The correlations between age and these derived measures were not statistically significant (see Table 
TABLE II. A/A and A/B thresholds (dB SPL) and derived measures of CDD and masking release (dB) due to onset asynchrony. Standard deviations for the mean data are shown in parentheses. The nine adults listeners $(\mathrm{A}-\mathrm{I})$ are shown in the top part of the table and the fifteen child listeners $(\mathrm{a}-\mathrm{O})$ are shown in the bottom part of the table. Age (years) is shown for children. The entry "NT" indicates incomplete data.

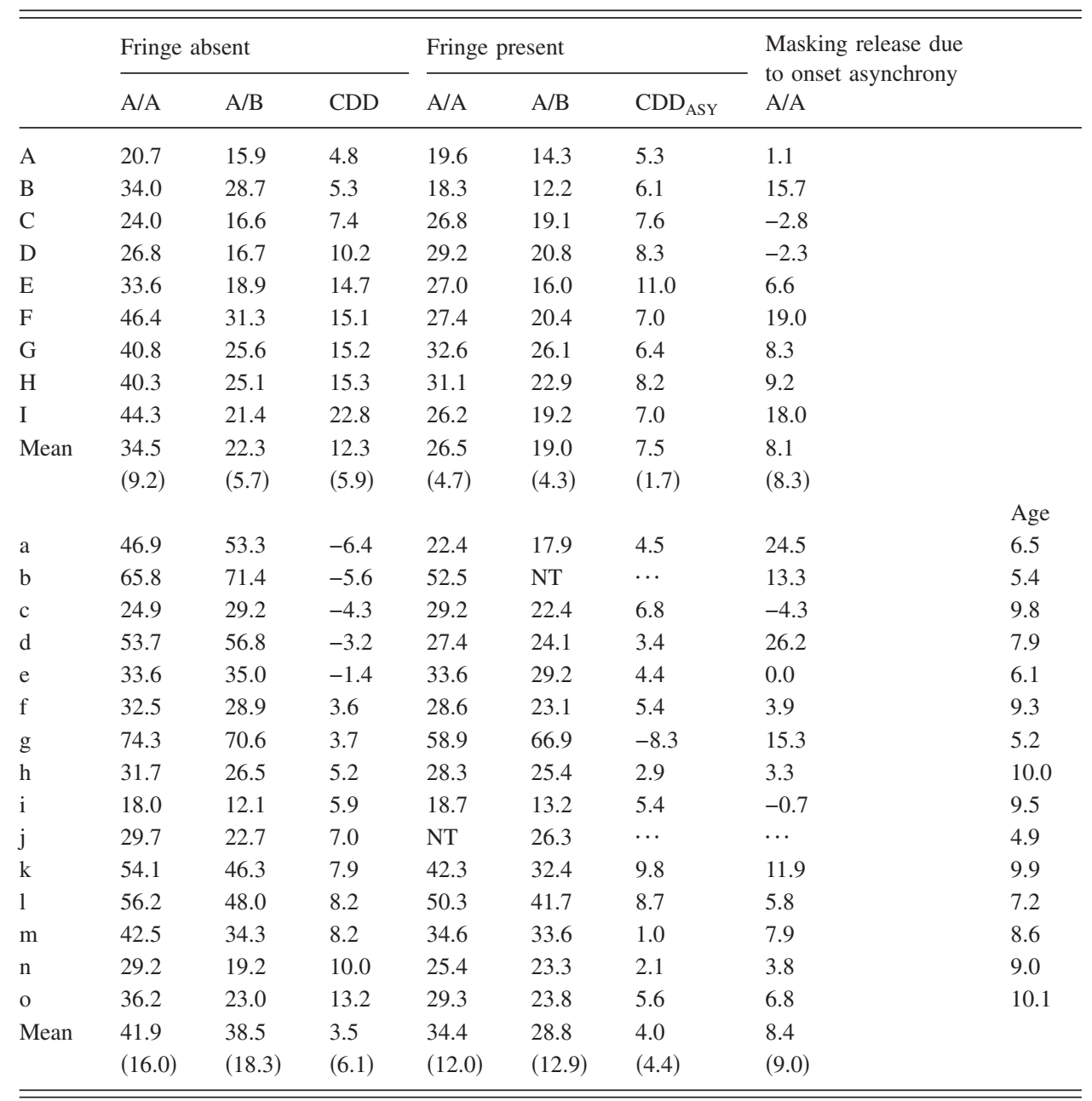

I), indicating that the neither the CDD nor the masking release due to onset asynchrony varied reliably with age in the present sample. Some of the correlations between age and masked thresholds were significant, however, indicating better performance with increasing age (see Table I). The correlation with age was significant for the $\mathrm{A} / \mathrm{B}$ condition, and approached significance for the A/A condition, using two tailed tests and a criterion probability of 0.05 . Table I also shows that the correlation with age was significant for the A/A condition where there was a temporal fringe.

The data of individual listeners are shown in Table II, and Fig. 1 summarizes the mean results for the conditions of this experiment. Inspection of Fig. 1 suggests that the children had higher masked thresholds than adults overall. This was tested with a repeated measures analysis of variance with the within subject factors of comodulation (A/A and A/B) and synchrony and the between subject factor of group. This analysis indicated that the children had significantly poorer thresholds than adults $\left(F_{1,20}=172 ; p<0.0001\right)$. The analysis also indicated significant effects of comodulation $\left(F_{1,20}=79 ; \quad p<0.0001\right)$ and synchrony $\left(F_{1,20}=12.1 ; p\right.$ $=0.002)$. The only interaction that was significant was between comodulation and group $\left(F_{1,20}=14.6 ; p=0.001\right)$. This

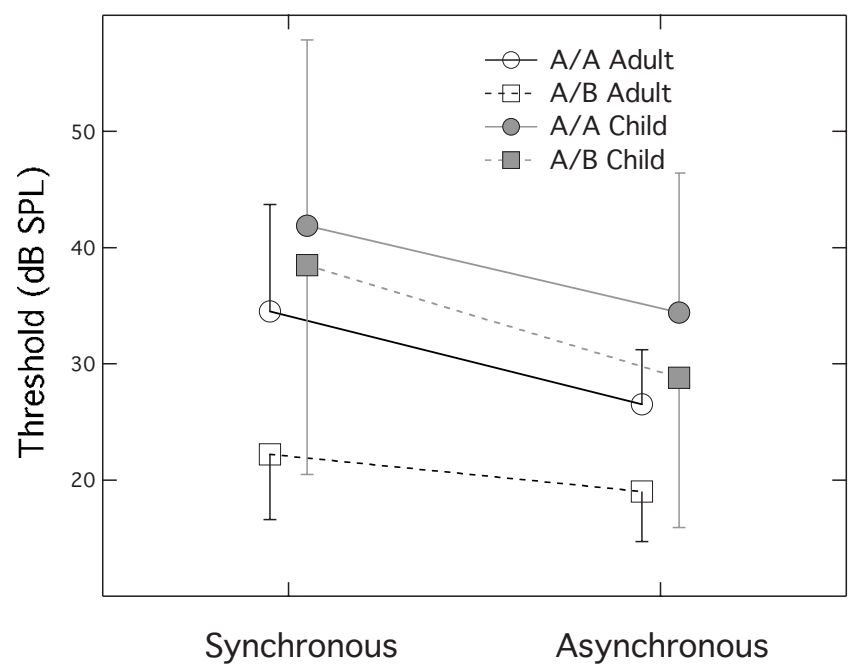

FIG. 1. Mean thresholds (dB SPL) for the synchronous and asynchronous masking conditions, plotted separately for the two masker conditions and observer groups, as indicated in the legend. Error bars show 1 s.d. (only the upper bar is shown for A/A conditions and only the lower bar is shown for A/B conditions for clarity). The data of the adults are depicted by the open symbols and the data of the children are depicted by the closed symbols. 
interaction reflects the fact that CDD was larger in adults than children, a finding that is examined more fully in the following sections.

\section{A. CDD and masking release resulting from signal/ masker onset asynchrony}

Because several aspects of the data of the adults appeared to be related to the magnitude of the CDD, the listeners are ordered alphabetically in Table II in terms of increasing CDD magnitude in order to aid discussion. In agreement with previous investigations of CDD in adults (e.g., McFadden and Wright, 1990; Hall et al., 2006), there was considerable variation in the masked threshold conditions as well as the derived CDDs. In adults, CDD was significant $\left(t_{8}=6.8\right.$; $p<0.001$ ), ranging from 4.8 to $22.8 \mathrm{~dB}$ with an average of $12.3 \mathrm{~dB}$; the masking release due to onset asynchrony in the A/A condition was significant $\left(t_{8}=2.9 ; p=0.02\right)$, ranging from -2.3 to $19.0 \mathrm{~dB}$ with an average of $8.1 \mathrm{~dB}$. In children, the CDD was significant $\left(t_{14}=2.17 ; p=0.047\right)$, ranging from -6.4 to $13.2 \mathrm{~dB}$ with an average of $3.5 \mathrm{~dB}$; the masking release due to onset asynchrony for the A/A stimuli was also significant $\quad\left(t_{13}=3.5 ; \quad p=0.004\right), \quad$ ranging from -4.3 to $24.5 \mathrm{~dB}$ with an average of $8.4 \mathrm{~dB}$. Thus the CDD and the release from masking resulting from onset asynchrony were significant in both adults and children. However, a t-test indicated that the average CDD of $3.5 \mathrm{~dB}$ in the children was smaller than the adult average of $12.3 \mathrm{~dB}\left(t_{22}\right.$ $=3.5 ; p=0.002$ ). The release from masking resulting from onset asynchrony did not differ significantly between adults and children $\left(t_{21}=0.32 ; p=0.93\right)$. This result suggests that children and adults were, on average, able to use the cue of onset asynchrony with comparable effectiveness.

The difference between the A/A and the A/B thresholds for the case where the masker and signal were gated asynchronously was also calculated. We will refer to this as $\mathrm{CDD}_{\mathrm{ASY}}$ to differentiate it from the more standard CDD where the masker and signal are gated on and off synchronously (see Table II). Whereas the adults showed an average CDD of $12.3 \mathrm{~dB}$, the average $\mathrm{CDD}_{\mathrm{ASY}}$ was $7.5 \mathrm{~dB}$, a reduction that was statistically significant $\left(t_{8}=2.6 ; p=0.03\right)$. The average $\mathrm{CDD}_{\mathrm{ASY}}$ for the children of $4.0 \mathrm{~dB}$ was similar to their average CDD of $3.5 \mathrm{~dB}$, and these values did not differ significantly $\left(t_{13}=0.053 ; p=0.96\right)$. The $\mathrm{CDD}_{\mathrm{ASY}}$ for the children was significantly smaller than that for the adults $\left(t_{20}\right.$ $=3.4 ; p=0.04$ ).

Although the above-mentioned analyses indicated several statistically significant differences between the results of the children and adults, it is important to note that there were large individual differences such that many children had results that were broadly within the adult range. For example, although the CDD of children was significantly smaller than that of the adults, the CDDs of six of the fifteen children were within 1 s.d. of the adult mean. Similarly, although the masked thresholds were higher in the children than in the adults, there was also considerable overlap between the groups for these thresholds (see Table II).

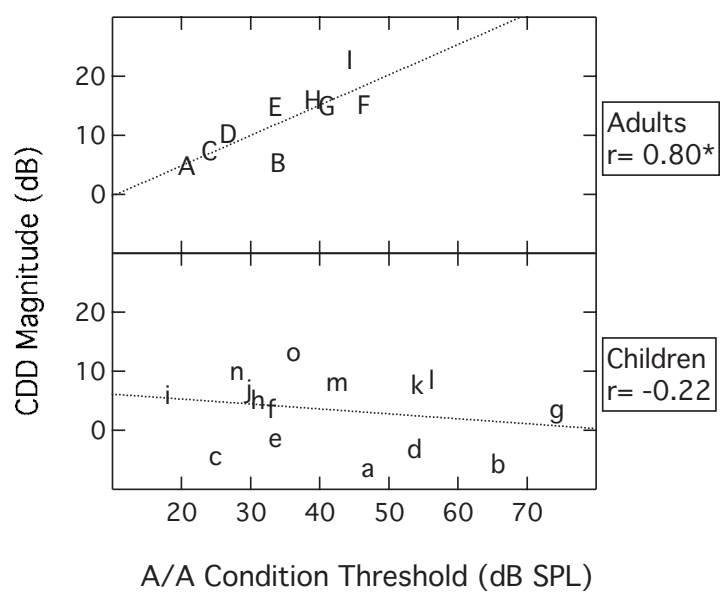

FIG. 2. The magnitude of CDD is plotted against the threshold in the A/A condition. The best-fitting regression line is also plotted. The data of the individual adults and children are plotted in the upper and lower panels, respectively. Correlations that are significant at the 0.05 probability criterion (two-tailed) are identified with an asterisk.

\section{B. CDD and perceptual fusion resulting from comodulation}

Several aspects of the results are pertinent to the issue of whether CDD is related to perceptual fusion resulting from comodulation. The top panel of Fig. 2 shows a plot of CDD against the threshold in the A/A condition for the adult listeners. As can be seen in Fig. 2, there was a tendency for the adults with high A/A thresholds also to show relatively high CDDs $(r=0.80 ; p=0.01)$. One interpretation of this significant correlation is that a factor contributing to high $\mathrm{A} / \mathrm{A}$ thresholds in some adult listeners is perceptual fusion of the signal and masker due to comodulation; the threshold improves in the $\mathrm{A} / \mathrm{B}$ condition because the signal and masker can be segregated when their modulation patterns are independent. Conversely, listeners with relatively low A/A thresholds do not have large effects related to perceptual fusion due to comodulation, and therefore do not show a large benefit when the segregation cue related to independent modulation is available in the A/B condition. Inspection of the bottom panel of Fig. 2 shows that, among children, the relation between $\mathrm{CDD}$ and the $\mathrm{A} / \mathrm{A}$ threshold was not significant $(r=-0.22 ; p=0.44)$. Possible reasons for this pattern of results are considered in Sec. IV.

As just discussed, the comparison of thresholds in the A/A condition with the magnitude of CDD may provide an indication of a release from perceptual fusion resulting from comodulation. Release from perceptual fusion can also result when a temporal fringe is introduced in the A/A condition. Figure 3 plots the masking release resulting from onset asynchrony in the A/A stimulus configuration. The top panel indicates a tendency for the adults with high A/A thresholds also to show relatively great making release due to onset asynchrony $(r=0.86 ; p=0.003)$. Together, the top panels of Figs. 2 and 3 suggest that adults with high A/A thresholds show improved performance with perceptual segregation cues based upon either across-frequency modulation pattern or onset asynchrony. This interpretation is also consistent with the data trends for CDD and masking release due to 


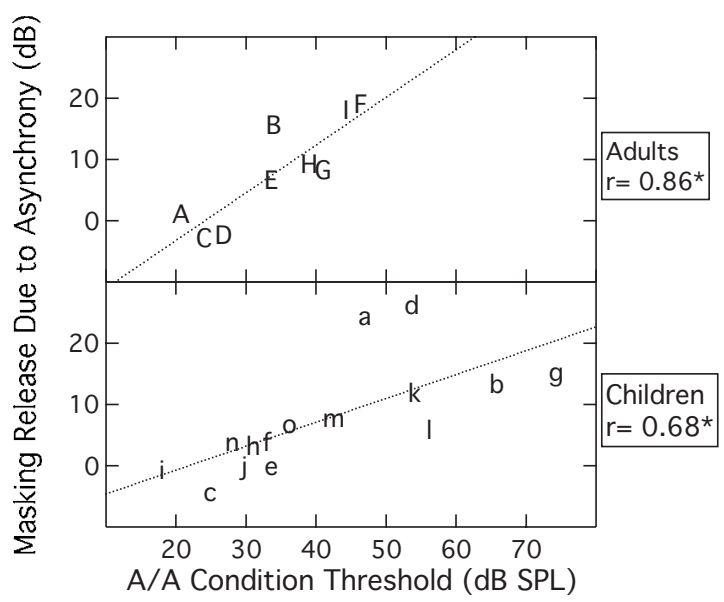

FIG. 3. The magnitude of the masking release resulting from onset asynchrony is plotted against the threshold in the A/A condition. The best-fitting regression line is also plotted. The data of the individual adults and children are plotted in the upper and lower panels, respectively. Correlations that are significant at the 0.05 probability criterion (two-tailed) are identified with an asterisk.

onset asynchrony reported in Hall et al. (2006). The bottom panel of Fig. 3 indicates that, among children, there was also a tendency for higher A/A thresholds to be associated with relatively great masking release due to onset asynchrony $(r$ $=0.68 ; p=0.007)$. That is, although high thresholds in the A/A masker condition were not associated with larger values of CDD (Fig. 2, bottom), they were associated with greater masking release due to asynchronous onset (Fig. 3, bottom). This suggests that high thresholds in the A/A conditions for children may not be related to fusion due to comodulation, but rather some other nonenergetic masking that can be reduced by onset asynchrony.

Recall that among adults, the $\mathrm{CDD}_{\mathrm{ASY}}$ was smaller than the CDD. The smaller $\mathrm{CDD}_{\mathrm{ASY}}$ is consistent with an interpretation that the CDD can result from both energetic masking and masking related to perceptual fusion, and that masking related to perceptual fusion is reduced under conditions of asynchronous gating. By this interpretation, the expectation is that adults showing higher A/A condition threshold values (and therefore relatively large effects related to perceptual fusion due to comodulation) should have a relatively great difference between $\mathrm{CDD}$ and $\mathrm{CDD}_{\mathrm{ASY}}$. In contrast, if children do not demonstrate a perceptual fusion effect related to comodulation, a strong association between the A/A condition threshold and the difference between CDD and $\mathrm{CDD}_{\mathrm{ASY}}$ is not expected. Figure 4 supports these expectations: Among the adults (top panel) high A/A condition thresholds were associated with a relatively great difference between $\mathrm{CDD}$ and $\mathrm{CDD}_{\mathrm{ASY}}(r=0.83 ; p=0.006)$; among the children, this was not the case $(r=0.22 ; p=0.44)$.

\section{GENERAL DISCUSSION}

Consistent with the previous study by Hall et al. (2006), the results of the present investigation indicated that adults with relatively high A/A thresholds were also likely to have relatively large CDDs. One interpretation of this result involves the idea that $\mathrm{CDD}$ (relatively worse performance in

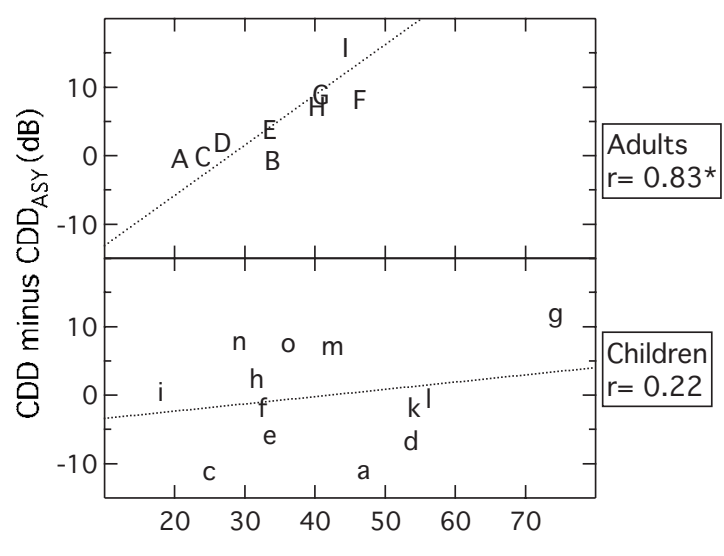

A/A Condition Threshold (dB SPL)

FIG. 4. The difference between $\mathrm{CDD}$ and $\mathrm{CDD}_{\mathrm{ASY}}\left(\mathrm{CDD}\right.$ minus $\left.\mathrm{CDD}_{\mathrm{ASY}}\right)$ is plotted against the threshold in the A/A condition. The best-fitting regression line is also plotted. The data of the individual adults and children are plotted in the upper and lower panels, respectively. Correlations that are significant at the 0.05 probability criterion (two-tailed) are identified with an asterisk.

the $\mathrm{A} / \mathrm{A}$ condition than in the $\mathrm{A} / \mathrm{B}$ condition) can arise from at least two factors: (1) a peripheral/energetic factor wherein comodulated flanking bands provide efficient masking and suppression of a comodulated signal; and (2) a central factor wherein perceptual fusion due to comodulation makes it difficult to separate the signal from the masker. By this interpretation, it is hypothesized that the adults showing the largest CDDs are those for whom the central, perceptual fusion factor has a relatively strong influence. A result that supported this interpretation was that adults with larger CDDs tended also to have higher thresholds in the A/A condition. There were several indications that, on average, children did not show a large CDD component related to perceptual fusion due to common modulation. For example, the CDD in children was relatively small in magnitude (although individual differences were noted), and there was no significant correlation between CDD and the threshold in the A/A condition. Overall, the results of the present study did not provide evidence that the A/A condition thresholds of the children tested were elevated due to perceptual fusion resulting from comodulation. This could occur if children were not very sensitive to comodulation as a perceptual fusion cue. It is possible that sensitivity to this grouping cue has a relatively protracted period of development. A related possibility is that high $\mathrm{A} / \mathrm{A}$ thresholds in children are dominated by factors other than the comodulation between the signal and masker. For example, children may be more likely than adults to have difficulty honing in on a frequency-specific target in the context of a complex background composed of multiple tones or narrow bands of noise. Consistent with this interpretation, Leibold and Neff (2007) found that children are more likely than adults to show informational masking not only under conditions of masker uncertainty, but also in conditions where a multiple-component maskers are fixed in frequency composition from trial to trial (as was the case in the present study). Such masking in the children of the present study could have overridden weaker effects related to comodulation. 
The present CDD results in children are relevant to previous developmental results obtained in a comodulation masking release (CMR) study (Hall et al., 1997). That study indicated that children were able to maintain CMRs similar to those of adults when two unique patterns of comodulation were carried by two separate sets of simultaneously present noise bands. One interpretation of this result was that children were as capable as adults in separating the two sets of noise bands by virtue of their different modulation patterns. This might seem inconsistent with an interpretation that the CDD of children is not influenced by perceptual fusion arising from comodulation. It is possible that this apparent inconsistency is related to stimulus factors. An important feature of the Hall et al. (1997) study is that the noise bands were presented continuously throughout a threshold run. This would give a listener extended opportunities to note comodulation among the various noise bands. In the synchronous conditions of the present study, the relatively brief nature of the gated stimuli would have limited the opportunity for the analysis of comodulation, and the common gating among the noise bands could have created a bias for grouping all of the noise components together. It is possible that children are less able to demonstrate grouping/segregation effects related to comodulation under such relatively challenging circumstances.

A finding of the present experiment that does not follow in a straightforward manner from the accounts considered thus far is that the adults showed a larger $\mathrm{CDD}_{\mathrm{ASY}}$ than the children. It seems reasonable to speculate that the $\mathrm{CDD}_{\mathrm{ASY}}$ of adults mostly reflects peripheral masking/suppression because masking related to more central auditory grouping cues should be largely overcome by cues related to onset asynchrony. If the $\mathrm{CDD}_{\mathrm{ASY}}$ is indeed peripherally driven, it is not clear why children would show a smaller $\mathrm{CDD}_{\mathrm{ASY}}$ than adults. This follows because there is evidence that peripheral auditory processing matures quite rapidly (Olsho, 1985; Abdala and Folsom, 1995). One possible interpretation is that despite a cue of onset asynchrony, a small part of the $\mathrm{CDD}_{\mathrm{ASY}}$ in adults is driven by a central effect related to grouping by comodulation.

\section{CONCLUSIONS}

(1) Children showed higher masked detection thresholds than adults, and there was a tendency for masked thresholds to improve with increasing age among the children.

(2) Both adults and children showed a significant CDD. Within the data of the children, there was no significant correlation between age and CDD, and CDD was smaller for children than for adults. The results were consistent with an interpretation that perceptual fusion arising from comodulation plays little or no role in the CDD of child observers. It was speculated that this could derive from poor sensitivity to comodulation as a perceptual fusion cue. Alternatively, a dominant informational masking effect, perhaps related to a difficulty in separating a frequency-specific target from a complex background, could override perceptual fusion effects related to comodulation. Although these interpretations apply to the average data, it was noted that there were large individual differences, with some of the children having CDDs within the adult range.

(3) Both adults and children showed a significant $\mathrm{CDD}_{\mathrm{ASY}}$. The $\mathrm{CDD}_{\mathrm{ASY}}$ was smaller than the CDD for the adults, but not for the children. The $\mathrm{CDD}_{\mathrm{ASY}}$ was smaller for the children than for the adults. It was suggested that even though the $\mathrm{CDD}_{\mathrm{ASY}}$ conditions involve onset asynchrony cues that reduce perceptual fusion between the signal and masker, perceptual fusion due to comodulation may nevertheless have a small contribution to the $\mathrm{CDD}_{\mathrm{ASY}}$ in the adult listeners. Such an effect could account for the finding that the $\mathrm{CDD}_{\mathrm{ASY}}$ was larger in the adults than in the children.

(4) Both adults and children showed masking release due to onset asynchrony. Among the children, this masking release was not significantly correlated with listener age. The magnitude of this masking release did not differ between adults and children, consistent with earlier findings indicating that the perceptual segregation cue of onset asynchrony shows a relatively early development.

\section{ACKNOWLEDGMENTS}

This work was supported by NIH NIDCD Grant No. R01 DC00418. Lori Leibold, Ruth Litovsky, and two anonymous reviewers provided helpful comments on a previous version of this manuscript. Madhu B. Dev and Lisa Whittle assisted in running subjects and supplied technical help.

Abdala, C., and Folsom, R. C. (1995). "The development of frequency resolution in humans as revealed by the auditory brain-stem response recorded with notched-noise masking," J. Acoust. Soc. Am. 98, 921-930.

ANSI (2004). "Specification for audiometers," ANSI S3.6-2004 (American National Standards Institute, New York).

Borrill, S. J., and Moore, B. C. (2002). "Evidence that comodulation detection differences depend on within-channel mechanisms," J. Acoust. Soc. Am. 111, 309-319.

Cohen, M. F., and Schubert, E. D. (1987). "The effect of cross-spectrum correlation on the detectability of a noise band," J. Acoust. Soc. Am. 81, 721-723.

Hall, J. W., Buss, E., and Grose, J. H. (2005). "Informational masking release in children and adults," J. Acoust. Soc. Am. 118, 1605-1613.

Hall, J. W., Buss, E., and Grose, J. H. (2006). "Comodulation detection differences for fixed-frequency and roved-frequency maskers," J. Acoust. Soc. Am. 119, 1021-1028.

Hall, J. W., Grose, J. H., and Dev, M. B. (1997). "Auditory development in complex tasks of comodulation masking release," J. Speech Lang. Hear. Res. 40, 946-954.

Kidd, G., Jr., Arbogast, T. L., Mason, C. R., and Walsh, M. (2002). "Informational masking in listeners with sensorineural hearing loss," J. Assoc. Res. Otolaryngol. 3, 107-119.

Kidd, G., Jr., Mason, C. R., Arbogast, T. L., Brungart, D. S., and Simpson, B. D. (2003). "Informational masking caused by contralateral stimulation," J. Acoust. Soc. Am. 113, 1594-1603.

Kidd, G., Jr., Mason, C. R., Deliwala, P. S., Woods, W. S., and Colburn, H. S. (1994). "Reducing informational masking by sound segregation," J. Acoust. Soc. Am. 95, 3475-3480.

Leek, M. R., Brown, M. E., and Dorman, M. F. (1991). "Informational masking and auditory attention," Percept. Psychophys. 50, 205-214.

Leibold, L. J., and Neff, D. L. (2007). "Effects of masker-spectral variability and masker fringes in children and adults," J. Acoust. Soc. Am. 121, 3666-3676.

Levitt, H. (1971). "Transformed up-down methods in psychoacoustics," J. Acoust. Soc. Am. 49, 467-477.

Lutfi, R. A. (1990). "How much masking is informational masking?" J. Acoust. Soc. Am. 88, 2607-2610. 
McFadden, D. (1987). "Comodulation detection differences using noiseband signals," J. Acoust. Soc. Am. 81, 1519-1527.

McFadden, D., and Wright, B. (1990). "Temporal decline of masking and comodulation detection differences," J. Acoust. Soc. Am. 88, 711-724.

Moore, B. C., and Borrill, S. J. (2002). "Tests of a within-channel account of comodulation detection differences," J. Acoust. Soc. Am. 112, 2099-2109.

Neff, D. L., and Callaghan, B. P. (1987). "Simultaneous masking by small numbers of sinusoids under conditions of uncertainty," in Auditory Processing of Complex Sounds, edited by W. A. Yost and C. S. Watson (Erlbaum, Hillside, NJ), pp. 37-46.

Neff, D. L., and Callaghan, B. P. (1988). "Effective properties of multicomponent simultaneous maskers under conditions of uncertainty," J. Acoust. Soc. Am. 83, 1833-1838.
Neff, D. L., and Green, D. M. (1987). "Masking produced by spectral uncertainty with multicomponent maskers," Percept. Psychophys. 41, 409415

Olsho, L. W. (1985). "Infant auditory perception: Tonal masking," Infant Behav. Dev. 8, 371-384.

Richards, V. M., Tang, Z., and Kidd, G. D., Jr. (2002). "Informational masking with small set sizes," J. Acoust. Soc. Am. 111, 1359-1366.

Turgeon, M., Bregman, A. S., and Ahad, P. A. (2002). "Rhythmic masking release: Contribution of cues for perceptual organization to the Crossspectral fusion of concurrent narrow-band noises," J. Acoust. Soc. Am. 111, 1819-1831.

Wright, B. A. (1990). "Comodulation detection differences with multiple signal bands," J. Acoust. Soc. Am. 87, 293-303. 